\title{
Macht ergreifen oder Macht verteilen?
}

Ein Aspekt, die noch immer nicht ausreichend untersucht wird, ist die Frage, was auf mittelfristige Sicht von dem Produkt kritischer soziokultureller Prozesse übrig bleibt, inklusive derer, die nicht fortbestehen. Möglicherweise sind es Lernerfahrungen zur Überwindung politischer Empörung jenseits der Protestplätze, die Ereignisse aus der Distanz miteinander verbinden können und über sichere Dienste wie Telegram geteilt werden können. Einst unvermeidbar scheinende Formen der räumlichen Eingrenzung und der kommunikativen Kontrolle können so außer Kraft gesetzt werden. Tausende von Normalbürgern verstehen heutzutage, wie man Nachrichten- und Videoportale aufbaut und sich in einer Gruppe zusammentut, um für seine Rechte einzustehen. Dafür werden »kollektive Aktionen« in einem Zusammenhang außerhalb der Parteien und den üblichen Mittlerorganisationen entwickelt, wie sie W.L. Bennett und A. Segenberg bezeichnen. Reguillo spricht von »losgelassenen Bürgern «, die misstrauisch gegenüber denjenigen werden, die sie $\mathrm{zu}$ vertreten behaupteten. Sie lernen jetzt, sich direkt und in einer Umgebung der Kontaktfreudigkeit auszudrücken: \#WeAreThe99\% oder \#YoSoy132. Die Hashtags sind, abgesehen von Werkzeugen zum gezielten Markieren von Informationen, zur Unterstützung bestimmter Personen oder Veranstaltungen und zum Teilen von Meinungen und Fotos, auch zu Räumen des Zusammentreffens geworden. In ihnen wird das Wir gebildet.

Soziale Bewegungen mit dem Ziel zur Filterung von Fake News und Fehlinformationen verdienen eine besondere Aufmerksamkeit und Anerkennung. Ein solches Projekt mit dem Namen Verificado19S wurde als Reaktion an das Erdbeben vom 17. September in Mexiko gestartet. Es hatte das Ziel, den unter Trümmern verschütteten Überlebenden zu helfen und Lebensmittel und Medikamente für sie zu sammeln. Dafür entwickelten die Verantwortlichen eine interaktive Karte mit zugehöriger Datenbank für die Überprüfung von Informationen aus den Medien und den sozialen Netzwerken, und um auf Bedürfnisse eingehen zu können, die ansonsten nicht gehört worden wären:

Wir koordinieren ein Vor-Ort-Team, das Informationen überprüft. Sobald sie bestätigt werden, erscheint auf der Karte eine Art Ebene mit verifizierten Infos. Wir teilen die Information, die uns geschickt wird mit anderen, und überprüfen sie auch nochmal selbst. Wir finden, dass die Überprüfung und Bestätigung von Informationen auf unserem Twitter-Account eine dringen- 
de Notwendigkeit für Mexiko-Stadt und andere Staaten darstellt (Beamonte 2017).

Ein Jahr darauf wurde Verificado 18 gegründet, ein unabhängiges gemeinsames Projekt aus der Initiative von Animal Político, +Español und Pop-Up Newsroom, mit dem Ziel der Aufdeckung unmöglich durchführbarer Wahlversprechen und manipulierter Inhalte im Zusammenhang der Wahlen in Mexiko. Zusätzlich zum Sammeln von Daten für die Aufdeckung unehrlicher Versprechen konnte man anhand der genutzten Methoden die Bedeutung der spezifischen Themen, der beteiligten Akteure und der angewandten Interaktionsmethoden genauer aufzeigen. Man bot eine WhatsApp-Hotline an, die es Nutzern möglich machte, zweifelhafte Informationen zur Überprüfung einzureichen. Ihre Seite verzeichnete mehr als 5 Millionen direkte Aufrufe, zu denen noch weitere Leser, Radiohörer und Fernsehpublikum hinzukamen, die über andere Medien von den Widerlegungen und Klarstellungen der Initiative erfuhren. Verificado 18 wurde von Firmen und Organisationen wie Facebook, Google News Initiative, Twitter, Open Society Foundation, Oxfam México und Mexicanos Contra la Corrupción y la Impunidad unterstützt. Ihr viermonatiges Wirken endete eine Woche nach den mexikanischen Wahlen vom 1. Juni 2018. Die Webseite blieb danach jedoch öffentlich und mit allen Inhalten erhalten.

Ähnliche Programme sind auch in anderen Ländern entstanden. Eine Vereinigung kolumbianischer Journalisten hat diese neue Tendenz als periodismo de confirmación, als Journalismus der Faktenprüfung, bezeichnet (colombiacheck.com). Gemeinsam haben sie eine Plattform organisiert, die richtige Information von falscher unterscheiden und den Wettbewerb zwischen zivilen und offiziellen, branchengesteuerten Medien verbessern soll.

Radikaler in der Infragestellung des E-Kapitalismus sind die so genannten Piratenparteien. Die erste wurde 2006 in Schweden gegründet, weitere folgten in Österreich, Dänemark, Finnland, Deutschland, Irland, den Niederlanden, Spanien und Polen. In Lateinamerika sticht der brasilianische Ableger dieser Partei hervor, der 2007 entstand. Ihre Hauptziele sind die Verteidigung des freien Informations- und Kulturflusses, die kooperative Arbeitsweise mit einem Maximum an Transparenz, und die Benutzung lizenzfreier Open-Source-Software, sowie die Förderung direkter Demokratie und der Solidarwirtschaft. Im Jahr 2010 gruppierten sich die Parteien übergreifend in der Pirate Parties International und bildeten 2012 auf europäischer Ebene die European Pirate Party. Sie erzielten 2011 bei den Wahlen zum Abgeordnetenhaus in Berlin 8,9\%, und stellten zwei Jahre später bereits 45 Landtagsabge- 
ordnete und 199 Stadtratsabgeordnete in Deutschland. In Schweden verzeichneten sie drei Jahre nach ihrer Entstehung 2011 bereits 215.000 Stimmen.

Trotz ihres Wachstums in rund 40 Ländern folgt ihre Reichweite einer instabilen Logik, die weit entfernt von den gewöhnlichen öffentlichen Auseinandersetzungen anderer Parteien ist. »Wir sind nicht hier, um an die Macht zu kommen«, behauptet Ásta Guthrún Helgadóttir, Piraten-Abgeordnete im isländischen Parlament, »sondern um die Macht zu verteilen« (Witte 2016).

Rodrigo Saturnino ist der brasilianische Parteiführer der Piraten und beobachtet das Phänomen aus wissenschaftlicher Perspektive. Er hat festgestellt, dass es keine »Piratenidentität « gibt, sondern eine »Piratenperspektive als Lebensentscheidung«. Vielmehr als um die Verteidigung spezifischer Ursachen gehe es um »eine Strategie des Widerstands« und man müsse »eine exotische Existenz im Feld der Parteienpolitik« bewahren (Saturnino 2016, 228-229). Seine »kontrapropagandistische« und ironische Ästhetik des Publizierens versucht sicherzustellen, dass die Demokratie »ein weniger undurchsichtiges und besser durchführbares Projekt« wird (Ibid., 233).

\section{Staatsbürgerschaft neu definiert}

Was gewinnen wir an den Schwankungen zwischen Erfolgen der zivilen Bewegungen, an der Zersetzung der Parteien und der Verherrlichung ihrer symbolischen Ziele? Was kommt nach den Veränderungen der politischen Kultur und der Formen ihrer Ausübung? Das Colectivo Situaciones schaffte es 2009 nach einer Auswertung der acht Jahre zurückliegenden massiven Aufstände vom Dezember 2001, die von Fernando de la Rúa geführte Regierung bis hin zu deren Auflösung unter Druck zu setzen und den Protest so weit zu intensivieren, bis ihrem Motto getreu »Haut Alle $\mathrm{ab}$ « waren (»Que se vayan todos! «). Das Kollektiv sagte aus, die massive Reaktion auf ökonomische Verdrängung sei »ausgehend von Fragmenten« zustande gekommen. Aus dieser Perspektive bedeutete seine kontrainformative Arbeit laut eigener Aussage die Entdeckung »einer einstimmigen und unbestimmten Willenskraft«. Dabei stell sich allerdings die Frage, wohin dieser unbestimmte Wille führen soll.

Wir beobachten heterogene Widerstände inmitten eines geschwächten und wenig glaubwürdigen Parteiensystems und materialistischen und symbolischen Wirtschaftsmodellen, die uns dort in Cluster einteilen, wo Proteste zerrinnen. Oft täuschen sie eine Teilnahme an der Debatte nur vor: Der Ausblick ist nicht besonders ermutigend, wenn wir ihn ausgehend von der 